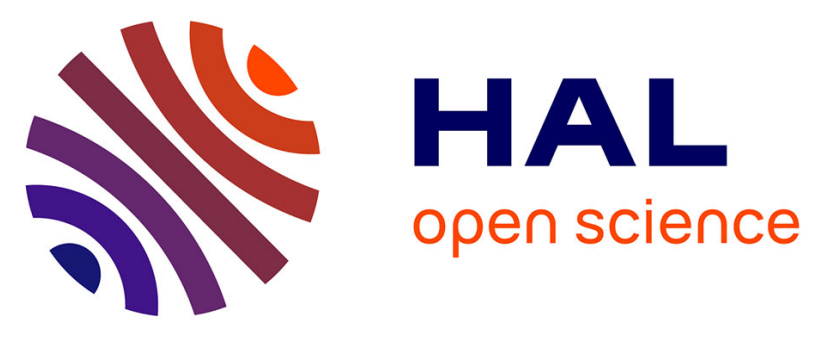

\title{
Predictive Maintenance Platform Based on Integrated Strategies for Increased Operating Life of Factories
}

\author{
Gökan May, Nikos Kyriakoulis, Konstantinos Apostolou, Sangje Cho,
} Konstantinos Grevenitis, Stefanos Kokkorikos, Jovana Milenkovic, Dimitris

Kiritsis

\section{To cite this version:}

Gökan May, Nikos Kyriakoulis, Konstantinos Apostolou, Sangje Cho, Konstantinos Grevenitis, et al.. Predictive Maintenance Platform Based on Integrated Strategies for Increased Operating Life of Factories. IFIP International Conference on Advances in Production Management Systems (APMS), Aug 2018, Seoul, South Korea. pp.279-287, 10.1007/978-3-319-99707-0_35 . hal-02177858

\section{HAL Id: hal-02177858 \\ https://hal.inria.fr/hal-02177858}

Submitted on 9 Jul 2019

HAL is a multi-disciplinary open access archive for the deposit and dissemination of scientific research documents, whether they are published or not. The documents may come from teaching and research institutions in France or abroad, or from public or private research centers.
L'archive ouverte pluridisciplinaire HAL, est destinée au dépôt et à la diffusion de documents scientifiques de niveau recherche, publiés ou non, émanant des établissements d'enseignement et de recherche français ou étrangers, des laboratoires publics ou privés.

\section{(c)(1)}

Distributed under a Creative Commons Attribution| 4.0 International License 


\title{
Predictive maintenance platform based on integrated strategies for increased operating life of factories
}

\author{
Gökan May ${ }^{1}$, Nikos Kyriakoulis ${ }^{2}$, Konstantinos Apostolou ${ }^{3}$, Sangje Cho ${ }^{1}$, Konstanti- \\ nos Grevenitis ${ }^{3}$, Stefanos Kokkorikos ${ }^{2}$, Jovana Milenkovic ${ }^{3}$, Dimitris Kiritsis ${ }^{1}$ \\ ${ }^{1}$ EPFL, ICT for Sustainable Manufacturing, EPFL SCI-STI-DK, Station 9, CH-1015 Lausanne, \\ Switzerland; ${ }^{2}$ Core Innovation and Technology O.E, Athens, Greece; ${ }^{3}$ ATLANTIS Engineering \\ SA, Thessaloniki, Greece \\ gokan.may@epfl.ch
}

\begin{abstract}
Process output and profitability of the operations are mainly determined by how the equipment is being used. The production planning, operations and machine maintenance influence the overall equipment effectiveness (OEE) of the machinery, resulting in more 'good parts' at the end of the day. The target of the predictive maintenance approaches in this respect is to increase efficiency and effectiveness by optimizing the way machines are being used and to decrease the costs of unplanned interventions for the customer. To this end, development of ad-hoc strategies and their seamless integration into predictive maintenance systems is envisaged to bring substantial advantages in terms of productivity and competitiveness enhancement for manufacturing systems, representing a leap towards the real implementation of the Industry 4.0 vision. Inspired by this challenge, the study provides an approach to develop a novel predictive maintenance platform capable of preventing unexpected-breakdowns based on integrated strategies for extending the operating life span of production systems. The approach and result in this article are based on the development and implementation in a large collaborative EU-funded H2020 research project entitled ZBre $4 k$, i.e. Strategies and predictive maintenance models wrapped around physical systems for zero-unexpected-breakdowns and increased operating life of factories.
\end{abstract}

Keywords: Industry 4.0, predictive maintenance, big data, asset management, smart factories, sustainable manufacturing, industrial production

\section{Introduction \& State-of-the-art}

The requirement of competitiveness is a constant objective of manufacturers [1]. For a successful shift toward Industry 4.0, companies must constantly innovate and implement new methods and approaches enabling them to reduce operating costs and increase the availability and reliability of their production equipment [2]. For this reason, any downtime due to technical issues with the equipment needs to be avoided and, if occurring, decreased as much as possible [3]. On that vein, maintenance in general and 
predictive maintenance strategies in particular, are now facing significant challenges to deal with the evolution of the equipment, instrumentation and manufacturing processes they should support. Preventive maintenance strategies designed for traditional highly repetitive and stable mass production processes based on predefined components and machine behaviour models are no longer valid and more predictive-prescriptive maintenance strategies are needed. The success of those adaptive and responsive maintenance strategies highly depends on real-time and operation-synchronous information from the production system, the production process and the individual product, which should enrich and extend more traditional techniques and models.

To meet the requirements mentioned above and aligned with the Industry 4.0 key objectives toward eco-factories of the future $[4,5]$, this study provides a holistic framework and a comprehensive set of integrated strategies encompassing the whole manufacturing line for addressing the issue of asset management in smart factories of industry 4.0 in order to extend the life of production systems. Doing so, the research aims at providing an answer as to what could be the proper strategies and associated technologies to effectively minimize downtimes of manufacturing systems. A large collaborative EU-funded H2020 research project entitled Z-Bre4k [6] has been the main driver of the described approach and is designed for its validation. The project consortium is formed by 17 organisations across Europe including industrial pilot plants, academic institutions and technology providing companies.

To this end, novel strategies are designed in this research to be deployed at the field in order to prevent/predict/diagnose/remediate failures, estimate remaining useful life (RUL) of assets, manage alarms and mitigation actions, and synchronise with shopfloor operations and plant management systems while ensuring the safety of workers. The ultimate aim is to introduce and apply a holistic approach via integrated strategies to increase maintainability, accurately predict the condition and the RUL of networked machines, and adapt the performance to increase the operating life span of production systems.

\section{Strategies for increased operating life of production systems}

The innovative synergies between online data gathering systems, real-time simulation models, data-based models and the knowledge management system form the main strategies which contribute to achieve zero breakdowns in manufacturing. In this context, the proposed solution comprises the introduction of eight (8) scalable strategies at component, machine and system level, all of which can be applied in the existing manufacturing plants with minimum interventions, targeting (1) the prediction occurrence of failure (Z-PREDICT), (2) the early detection of current or emerging failure (ZDIAGNOSE), (3) the prevention of failure occurrence, building up, or even propagation in the production system (Z-PREVENT), (4) the estimation of the RUL of assets (Z-ESTIMATE), (5) the management of the aforementioned strategies through event modelling, KPI monitoring and real-time decision support (Z-MANAGE), (6) the replacement, reconfiguration, re-use, retirement, and recycling of components/assets (ZREMEDIATE), (7) synchronizing remedy actions, production planning and logistics 
(Z-SYNCHRONISE), (8) preserving the safety, health, and comfort of the workers (ZSAFETY). Each of the developed strategies are triggered based on predicting, detecting and assessing the impact of system level events that cause low performances, generate failures, and increase the costs. Figure 1 highlights the synergies and interactions between the eight Z-Strategies for building a novel predictive maintenance platform and the role of each strategy is further explained below.

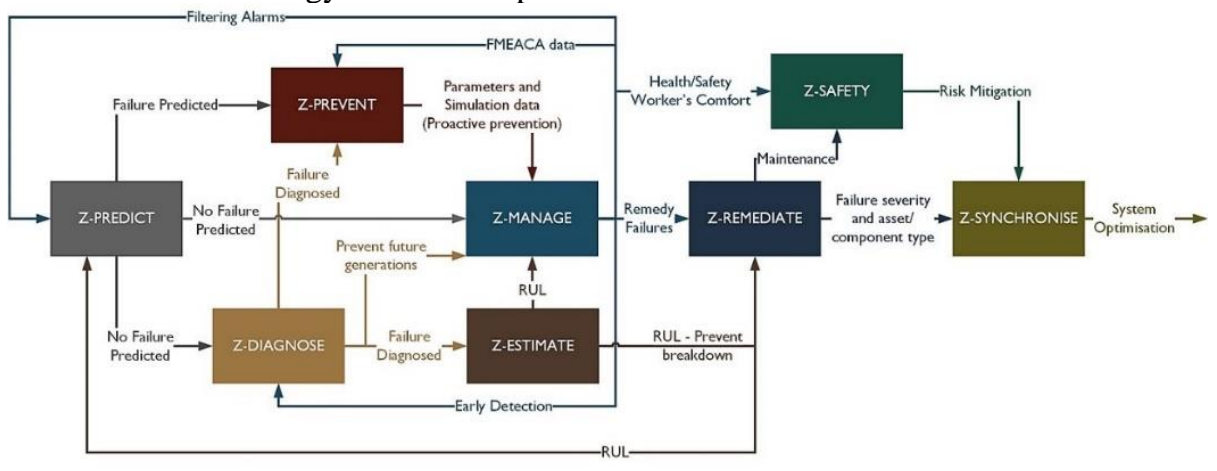

Fig. 1. Synergies and interactions between the eight Z-Strategies

Z-PREDICT: The events detected from the physical layer of the system are engineered into high value data that stipulates new and more accurate process models. Such an unbiased systems behaviour monitoring and analysis provides the basis for enriching the existing knowledge of the system (experience) learning new patterns, raising attention towards behaviour that cause operational and functional discrepancies (e.g. alarms for predicted failures) and the general trends in the shop-floor. The more the data pool is being increased the more precise (repeatability) and accurate the predictions will be. The estimations for the future states involve the whole production line - network of machines and components. The system can thus predict with high confidence the expected performance of components and their maintenance needs, predicting current or emerging failures, allowing better production planning and decision making on their RUL. Hence, the ability to optimise the manufacturing processes according to the RUL, production needs, and the maintenance operations is the key innovation to fulfil the industrial requirements.

Z-PREVENT: The prevention of failure occurrence strategy is based on the prediction strategy (i.e. degraded performance of assets or failure) realised across the shopfloor for condition monitoring of machinery and respective produced quality. The ZPREDICT is predecessor of Z-PREVENT. The initial estimation of the future states is based on the simulation and modelling of the parameters. For each predicted failure or low performance (e.g. due to fatigue, wear), the responsible factors are identified and flagged through the FMEA system. The system analyses these factors based on an initial estimation, which after the simulation these are updated recursively. The result of this process is to avoid the building up or even propagation of a failure that leads to breakdown based on each recorded event both from previous and current states. The strategy thus prevents multiple alarm activations on similar failures. 
Z-DIAGNOSE: This strategy is invoked when a current or an emerging failure is detected considering the condition at all three levels - machine, product, shop-floor. In such a scenario, an alarm is being triggered to flag the events that resulted in a failure or system performance degradation. By mapping the true reasons, the system is then able to avoid generating the failure or its emergence by weighting the system model. The strategy also involves more actions and processes to deal both with the generation of the diagnosed failure, and its severity increase to the next iterations as well as its impact to the production line. Depending on the criticality of the generated failure, the system can either adapt its parameters to prolong the RUL until the next maintenance, or plan to the production for maintenance. The final decision on the actions is based on the Z-MANAGE strategy.

Z-ESTIMATE: This strategy combines the information from the Z-DIAGNOSE and Z-PREDICT estimating the RUL of the assets. The estimated values are also combined with the information from the maintenance operations (physical examination from operators) as well as from the specifications provided from the manufacturer. The latter is used as the starting point for the estimation process, which after each iteration the deviation of the real-model from the physical model is reduced having an accurate virtual-model wrapped around the actual state of each machine and its components. The trends for the fatigue and wear rates provide a confident RUL estimation.

Z-MANAGE: This strategy is executing the overall supervision and optimisation of the system. The failures are processed with the Decision Support System (DSS) tools and are interfaced with Manufacturing Execution Systems (MES). False positives and false negatives are clustered within the Z-PREDICT and Z-PREVENT Strategies. To achieve so, the previous acquired knowledge and incidents are also processed to fine tune the system's performance. Additionally, the production is optimised by better scheduling (Z-SYNCHRONISE), taking into account the impact of each failure. The optimised scheduling and adaptability of the manufacturing improves the overall flexibility, placing a premium on the production systems, extending their operating life, while preserve increased machinery availability.

Z-REMEDIATE: This strategy involves the decision making in the event of a failure, which classifies and categorises the input in terms of criticality, type, etc. Based on the component/assets types (repairable-non repairable) and their RUL the strategy decides for the following: (1) replace, (2) reconfigure and/or re-use, (3) retire, and (4) recycle. This strategy triggers the Z-SYNCHRONISE and Z-SAFETY strategies from which the maintenance actions can be planned and organized.

Z-SYNCHRONISE: The predecessor Z-REMEDIATE strategy identifies the type of action required for diagnosed failures which are then fused with the Z-MANAGE output. This strategy synchronises all the remedy actions with internal and external supply-chain tiers, as well as with production planning and logistics. It is therefore responsible to shift the production from one machine to another due to failure or deteriorated condition/performance, acting as the "end-effector" thus leading to optimised scheduling and reduced costs by carrying out maintenance activities on time.

Z-SAFETY: This strategy is invoked to increased Health \& Safety during Z-Bre4k shop-floor operations. Since most of the accidents occur during maintenance actions, the Z-SAFETY prevents any activation to the machine that is under investigation or 
repair. The "Safety-Mode" lifts any unauthorised control from the personnel for the whole duration of the maintenance. Apart from reducing the accidents Z-SAFETY also takes into account the comfort of the human personnel on the shop-floor, e.g. extreme heat or noise may be tolerable for the machines but not for humans. Therefore, the health \& safety procedures are also taken into account towards the operation feedback of the whole production line.

\section{$3 \quad$ Predictive maintenance platform based on Z-Strategies}

Manufacturing enterprises are pushed to take local actions: thinking globally however staying economically compatible within the local context. In order to achieve high precision manufacturing of complex products, there has to be a fundamental rethink on how to improve the operation of machines and improved controls. The improvement should not only concern the individual machines as isolated islands but encompass the totality of production process as a system of interrelated elements that seek to maximise efficiency, productivity, customer satisfaction; whilst at the same time eliminating waste and excess inventory. For that purpose, aligned with the Z-Strategies, a set of technologies and overall system architecture have been identified as a part of the proposed approach, following the method and procedures developed and proposed by May et al. (2017) [7].

The first high-level description to lead to the definition of the predictive maintenance platform consisted in identifying and classifying all components that can be called as the tools' landscape and logical architecture, i.e. conceptual view. Figure 2 presents this landscape by proposing a compact representation of the involved tools.

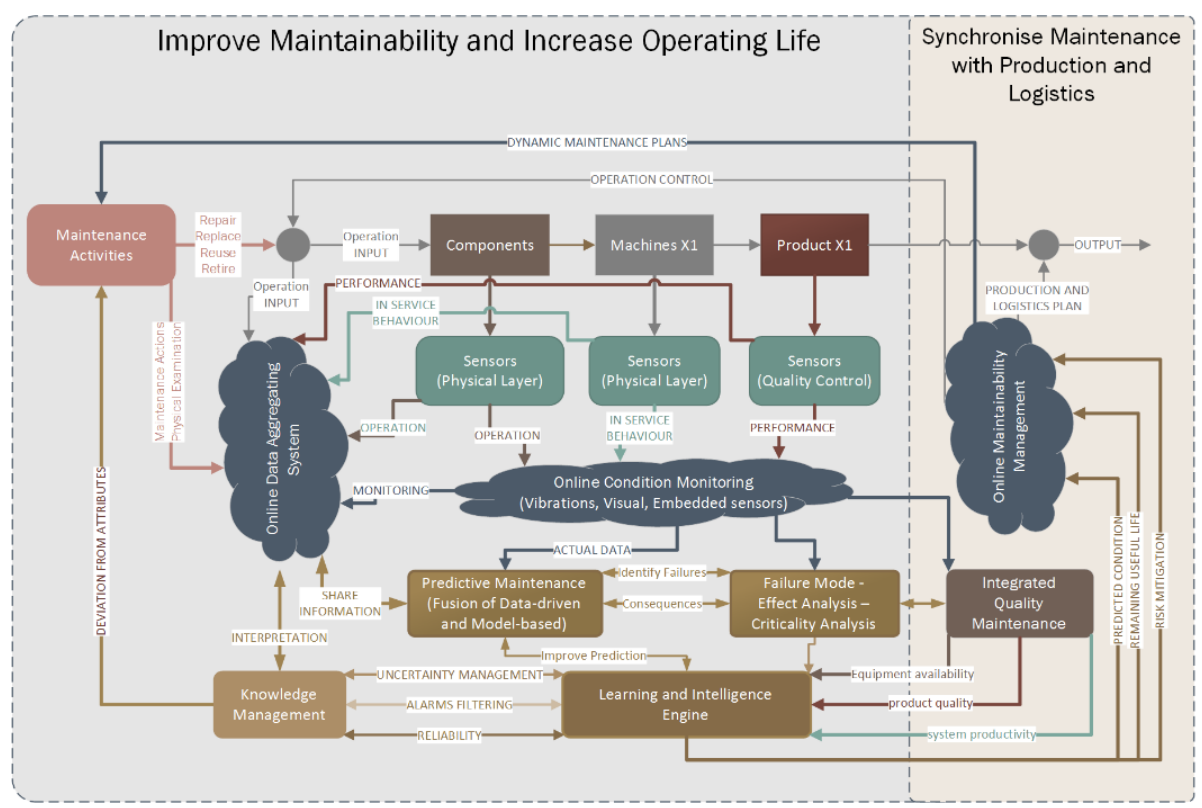

Fig. 2. Tools' landscape and logical architecture of the predictive maintenance platform 
Based on the proposed approach and defined conceptual view of the system, in ZBre $4 \mathrm{k}$ a novel predictive maintenance platform will be developed and demonstrated in three pilot plans proving its universal applicability for the achievement of zero breakdowns in manufacturing. Therefore, the predictive maintenance platform will:

- Introduce a novel design for predictive maintenance based on three levels: machine (network of components), product, and shop-floor (network of machines). It will reconfigure the system to increase its performance (shorter cycles), increase its quality, and its availability by the employment of eight strategies to maximise these factors.

- Make accurate predictions for the future states of the components/machines/systems by the employment of intelligent and adaptive simulators forecasting the generation of failures, the fatigue and wear levels, estimating the RUL triggering respective remedy actions. The condition monitoring will provide data about the actual status which will update the simulation results, increasing its accuracy. The DSS will synchronise the plans for maintenance, production and logistics.

- Estimate the RUL through its simulation capabilities, calling for maintenance and suggesting the optimal times to place orders for spare parts, reducing the related costs. The increased predictability of the system and the failure prevention actions will reduce the number of failures, maximise the performance, decrease the repair/recover times reducing further the costs.

- Optimise the performance of the machines, based on the current and predicted fatigue/wear levels allowing actions to maintain and increase the operating life of these assets, as well as to reduce the unexpected failures and breakdowns.

Following the development of the conceptual view, the required components have been highlighted in a preliminary architectural view, identifying services and dependencies within the Z-Bre4k platform. Later, new components were added in order to cover all the required functionalities of the resulting predictive maintenance platform. As a result, definitions, identifications and classification of the system principle and its process is presented in Figure 3. Besides the defined overall architecture, Table 1 presents initial links on how these strategies are integrated within the overall architecture and how they are associated with each component of Z-Bre4k platform.

Z-Bre4k components, their functionality and their interactions are thus described in the overall architecture (Figure 3). Initial data, generated by shop-floor assets (i.e. sensors, cameras, industrial/IoT devices, etc.) is collected by Condition Monitoring, Cognitive Embedded Condition Monitoring and Machine Simulators components. All data is sent to the Industrial Data Spaces (IDS) reaching the overall Z-Bre4k platform after which they are homogenized by the Semantic Framework and used by the components in a unified manner. Within the platform, users/employees are using HMI in order to add data and/or parameterize components. Specifically, FMECA (Failure mode, effects, and criticality analysis) component needs these inputs to calculate risks, Risk Priority Numbers (RPNs), criticality matrices and alerts in order to send data to DSS, that further generates and delivers strategies, recommendations, notifications, reports 
and updated schedules. Finally, M3 Gage, M3 Software and VRfx components are related to XYZ cloud points, 3D representations and visualization data of physical objects.

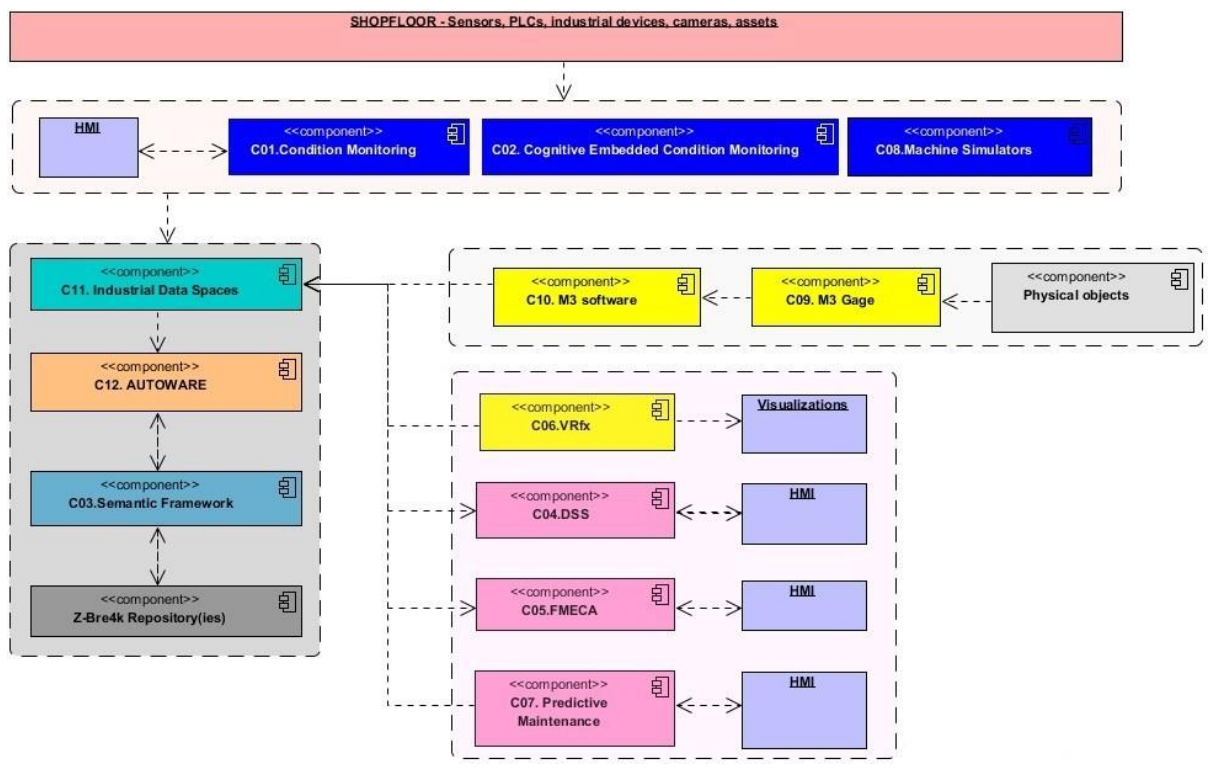

Fig. 3. Overall architecture of the predictive maintenance platform

Table 1. Z-Strategy and component association

\begin{tabular}{|c|l|l|}
\hline No. & Z-Strategy & Components \\
\hline $\mathbf{1}$ & Z-PREDICT & $\begin{array}{l}\text { Condition monitoring, } \\
\text { Machine simulators, VRfx, Predictive Maintenance. }\end{array}$ \\
\hline $\mathbf{2}$ & Z-PREVENT & Machine simulators, Predictive Maintenance, FMECA. \\
\hline $\mathbf{3}$ & Z-DIAGNOSE & Predictive Maintenance, FMECA. \\
\hline $\mathbf{4}$ & Z-ESTIMATE & Machine simulators, VRfx, Predictive Maintenance. \\
\hline $\mathbf{5}$ & Z-MANAGE & Predictive Maintenance, FMECA, DSS. \\
\hline $\mathbf{6}$ & Z-REMEDIATE & FMECA, DSS. \\
\hline $\mathbf{7}$ & Z-SYNCHRONIZE & AUTOWARE Communication Middleware. \\
\hline $\mathbf{8}$ & Z-SAFETY & DSS. \\
\hline
\end{tabular}

The resulting Z-Bre4k system will be demonstrated in three key sectors with the strongest SME presence (i.e. automotive, food and beverage, consumer electronics) for a wide range of components and machines with different operational requirements and behaviours, illustrating the potential and full value of Z-Bre4k as a holistic framework to address predictive maintenance strategies for operation in high diversity of machinery (e.g. robotic systems, inline quality control equipment, injection moulding, stamping press, high performance smart tooling/dies and fixtures), including highly challenging and sometimes critical manufacturing processes (e.g. automated packaging industry, multi-stage zero-defect adaptive manufacturing of structural light-weight component for automotive industry, short-batch mass customised production process for consumer electronics and health sector). 


\section{Discussion and concluding remarks}

The main goal of the predictive maintenance approach and implementation is to provide machine builders and designers (OEMs), industrial component suppliers and engineering software developers with novel solutions which will: (1) improve the performance of the manufacturing processes; (2) increase the machine maintainability; (3) provide predictions on damages/failures; (4) understand and interpret the source of the failures enhancing eventually the design process; (5) increase the availability of the machine builders while making them cost effective; and thus (6) increase OEE.

In this context, the study will: (a) act as a comprehensive and practical guide for optimizing production machineries and processes by implementing predictive maintenance principles; (b) transform machine tool and process related data into useful information that could support machinery prognosis and optimization strategies by enabling model-based control of machine tools based on actual machine life-cycle parameters.

Accordingly, the expected impacts are highlighted as follows: (1) improved predictive maintenance and system adaptability for manufacturing systems and processes; (2) new maintainability concepts based on predictive maintenance with improved machine reliability (MTBF) and reduced maintenance costs; and (3) incorporating intelligent systems and data analysis methods for achieving smart factories of Industry 4.0.

Future work will focus on implementing and validating the proposed approach on several use cases in different industries, demonstrating its ability to support major actors of the manufacturing sector to take advantage of the digital transformation.

Acknowledgements. This work has been carried out in the framework of Z-Bre4k Project, which has received funding from the European Union's Horizon 2020 research and innovation programme under grant agreement $\mathrm{N}^{\circ} 768869$.

\section{References}

1. Swanson, L.: Linking maintenance strategies to performance. International Journal of Production Economics 70(3), 237-244 (2001)

2. Wang, S., Wan, J., Li, D., \& Zhang, C.: Implementing Smart Factory of Industrie 4.0: An Outlook. International Journal of Distributed Sensor Networks, 12(1), 3159805 (2016).

3. Lee, J., Kao, H.-A., \& Yang, S.: Service Innovation and Smart Analytics for Industry 4.0 and Big Data Environment. Procedia CIRP 16, 3-8 (2014).

4. Lee, J., Bagheri, B., \& Kao, H.-A.: A Cyber-Physical Systems architecture for Industry 4.0based manufacturing systems. Manufacturing Letters 3, 18-23 (2015).

5. May, G., Stahl, B., Taisch, M.: Energy management in manufacturing: Toward eco-factories of the future - A focus group study. Applied Energy 164, 628-638 (2016).

6. Z-Bre4k Project Homepage, https://www.z-bre4k.eu, last accessed 2018/03/21.

7. May, G., Ioannidis, D., Metaxa, I. N., Tzovaras, D., \& Kiritsis, D.: An Approach to Development of System Architecture in Large Collaborative Projects. IFIP Advances in Information and Communication Technology, 67-75 (2017) 